\title{
HEMORRAGIA SUBARACNÓIDEA PERIMESENCEFÁLICA NÃO-ANEURISMÁTICA
}

\author{
APRESENTAÇÃO DE CASO E REVISÃO DE LITERATURA
}

\author{
MÁRIO H. TSUBOUCHI*, WALTER O. ARRUDA*, RICARDO RAMINA **, MURILO S. MENESES **, \\ ARI A. PEDROZO**, LUIZ F. FAVORITO***, ROGÉRIO S. CLEMENTE***
}

\begin{abstract}
RESUMO - Os autores apresentam um caso de hemorragia subaracnóidea perimesencefálica nāo aneurismática em um paciente masculino de 42 anos, com excelente evoluçāo clínica. É realizada revisāo da literatura, sendo apresentados aspectos clínicos e neurorradiológicos desta entidade benigna.
\end{abstract}

PALAVRAS-CHAVES: hemorragia subaracnóidea não-aneurismática, aneurisma cerebral, acidente vascular cerebral hemorrágico, hemorragia subaracnóidea perimesencefálica.

\section{Nonaneurysmatic perimesencephalic hemorrhage: a case report and review of the literature}

SUMMARY - A 42 year-old man with subarachnoid hemorrhage was admitted in our Service (Hunt \& Hess grade II). CT-scan disclosed predominantly perimesencephalic subarachnoid hemorrhage. Digital subtraction angiographic study did not disclose either cerebral aneurysm or any other potential site of intracranial bleeding. The evolution was uneventful. A DSA was repeated one month later and was negative. Four months later, the patient was asymptomatic. The authors review the literature and emphasize the importance in recognizing this benign clinical form of nonaneurysmatic subarachnoid hemorrhage. hemorrhage.

KEY-WORDS: nonaneurysmatic perimesencephalic hemorrhage, cerebral aneurysm, subarachnoid

A maioria das hemorragias subaracnóideas espontâneas sāo decorrentes de rotura de aneurismas intracranianos (70-80\%) ou de malformaçōes arteriovenosas (5-10\%), constituindo entidade clínica com elevados riscos de sequelas neurológicas e mortes ${ }^{5,6,17,18,21}$. Em aproximadamente $15 \%$ dos pacientes com hemorragia subaracnóidea a causa do sangramento é indeterminada, nāo se observando qualquer anormalidade ao exame arteriográfico cerebral ${ }^{23,8-11,16}$. Dentro desse grupo de casos tem se delineado um quadro mais específico chamado "hemorragia perimesencefálica não aneurismática"(HPNA), com prognóstico excelente ${ }^{19}$.

Os autores relatam neste estudo um caso dessa singular forma de acidente vascular cerebral hemorrágico e tecem comentários sobre ela.

\section{RELATO DE CASO}

DPSC, masculino, branco, 42 anos de idade; profissão: relojoeiro. Foi internado no Hospital das Naçōes com história de há 5 dias ter apresentado quadro súbito de cefaléia holocrânica intensa, náuseas, vômitos e tontura. Negava perda de consciência ou crises epiléticas. Exame físico geral normal. Exame neurológico revelava um paciente

Unidade de Ciências Neurológicas, Hospital das Naçōes, Curitiba: *Neurologista; **Neurocirurgião; ***Especializando em Neurocirurgia. Estudo apresentado como Tema Livre no XV Congresso Brasileiro de Neurologia (Porto Alegre, RS, 1992). Aceite: 20-julho-1993.

Dr. Mário H. Tsuboushi - Rua Raphael Papa 10 - 82530-190 Curitiba PR - Brasil. 


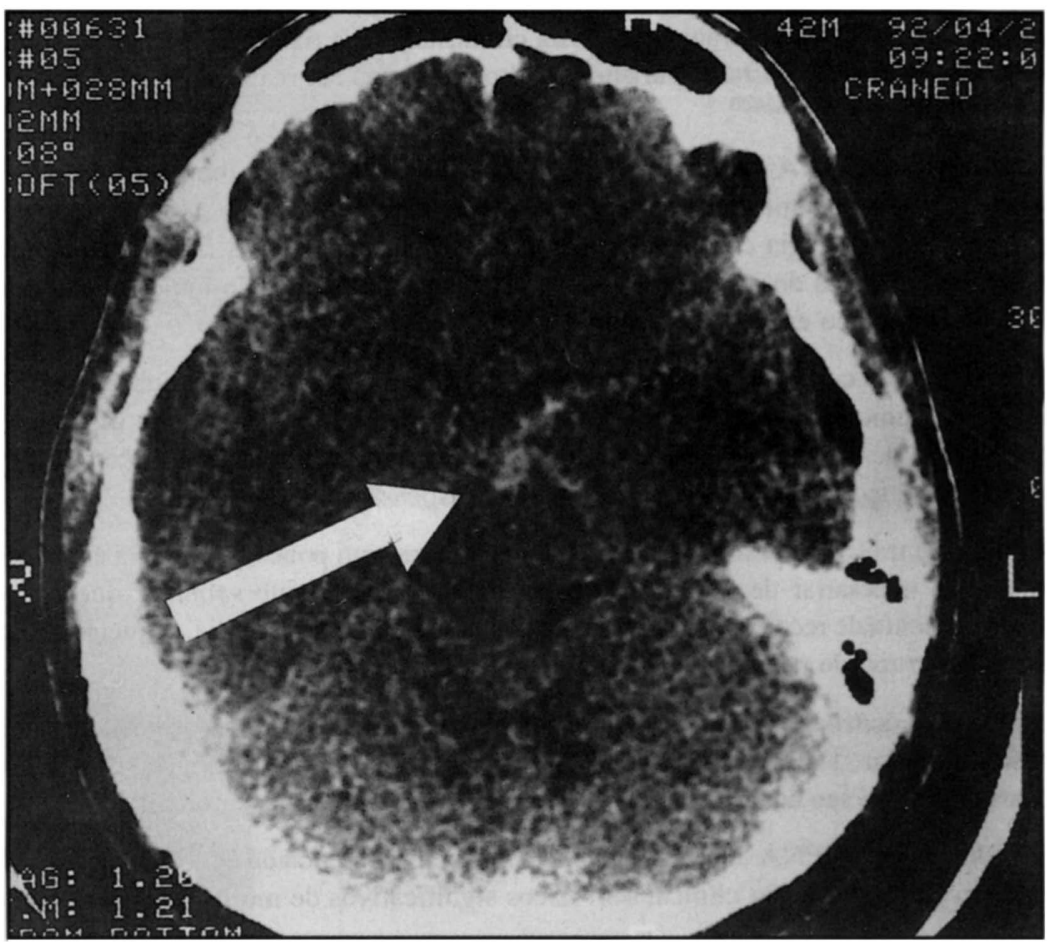

Figura 1. Paciente DPSC. Tomografia computadorizada de crânio (sem contraste) mostrando a presença de hemorragia subaracnóidea na cisterna interpeduncular.

em grau II de Hunt \& Hess (cefaléia, rigidez de nuca, sem déficits neurológicos focais). Trazia uma tomografia computadorizada de crânio (TC) em que se evidenciava hemorragia subaracnóidea mais acentuada na regiáo da fossa interpeduncular (Figura 1). Exames complementares (hemograma, dosagens séricas de sódio, potássio, uréia, creatinina, glicose, tempo de ativaçâo da protrombina, tempo de tromboplastina parcial ativada) foram normais. Foi realizada angiografia digital cerebral que não revelou aneurisma ou outra malformação arteriovenosa, nem vasoespasmo. $O$ tratamento consistiu de repouso no leito, analgésicos, anti-eméticos e nimodipina ( $60 \mathrm{mg}$ via oral $4 /$ 4 horas por 21 dias). O paciente evoluiu sem intercorrências, recebendo alta hospitalar assintomático (no quarto dia após o internamento). Um mês após a alta, foi submetido a novo estudo angiográfico, que se revelou normal. $\mathrm{Na}$ última avaliaçāo ambulatorial, 4 meses após o evento, apresentava-se bem, sem queixas, tendo retornado às suas atividades diárias normais.

\section{COMENTÁRIOS}

A "hemorragia subaracnóidea de etiologia indeterminada"(HSEI), cujo estudo angiográfico nāo demonstra aneurismas cerebrais ou outras fontes intracranianas de sangramento, representa um grupo heterogêneo de acidentes vasculares cerebrais hemorrágicos com evoluçāo clínica e prognóstico variáveis. Estudos que năo diferenciavam subgrupos nessa populaçăo referem risco de ressangramento e de isquemia cerebral por vasoespasmo na incidência de pelo menos $10 \%$ para cada uma dessas complicaçōes $24,7,9,11$.

Desde 1985, van Gijn e colaboradores ${ }^{18-20}$, após analisarem o padrāo de hemorragia no espaço subaracnóide pela TC, vêm definindo duas formas bem distintas de HSEI: 1. tipo perimesencefálico, em que o sangramento se localiza predominantemente ou somente nas cisternas em torno do mesencéfalo (cisternas interpeduncular, ambiens e quadrigeminal) sem extensão para as fissuras inter-hemisféricas e laterais, parênquima cerebral ou sistema ventricular; 2. tipo aneurismático, em que a distribuiçāo da hemorragia subaracnóidea é indistinguível da habitualmente observada 
nas roturas de aneurismas intracranianos. Nesta circunstância, a repetição do estudo angiográfico torna-se necessária para descartar aneurismas ocultos, devendo ser realizada 4-6 semanas após um primeiro estudo negativo ${ }^{12-14,18-20}$.

Pacientes com HPNA geralmente não perdem a consciência no momento do ictus, não se apresentam criticamente enfermos, e os sinais de irritaçāo meníngea são os únicos achados neurológicos ${ }^{12-14}$. Hemorragia confinada às cisternas perimesencefálicas fica demonstrada na TC obtida dentro de 72 horas do evento, pois além deste período ocorre diminuiçāo da sensibilidade em se detectar sangue no espaço subaracnóideo ${ }^{1,820}$.

A angiografia cerebral é obrigatória na avaliação inicial, desde que existe sempre a possibilidade da hemorragia perimesencefálica ter sido decorrente da rotura de um aneurisma da artéria basilar ${ }^{19}$ ou de outros vasos das proximidades. Sendo o estudo angiográfico negativo e sem evidências de vasoespasmo, novo exame poderá ser dispensável.

A única complicação descrita na HPNA e que ocorre com pouca frequência é a hidrocefalia, podendo ou nāo necessitar de derivaçāo ventricular ${ }^{15}$. É importante salientar que nāo existem relatos até o momento de recorrência da hemorragia, isquemia cerebral tardia, sequelas neurológicas ou mortes neste grupo de pacientes ${ }^{12,1419}$.

Especula-se, com base nas características clínicase tomográficas, que a origem do sangramento seja venoso ou capilar. Tal hipótese necessita ainda de comprovaçăo patológica, difícil de obter-se nesses casos devido ao seu curso benigno e à inexistência de estudos postmortem ${ }^{2,1214,19}$.

Em conclusāo, a HPNA deve ser reconhecida e bem diferenciada dentro do grupo das HSEI, pois possui excelente evolução clínica, sem riscos significativos de morbidade ou mortalidade.

\section{REFERÊNCIAS}

1. Adams HP Jr, Kassel NF, Torner JC, Sahs AL. Computed tomographic and clinical correlations in recent aneurysmal subarachoid hemorrhage: a preliminary report of the Cooperative Aneurysm Study. Neurology 1983, 33: 981-988. 2.Adams HP, Gordon DL. Nonaneurysmal subarachnoid hemorrhage. Ann Neurol 1991, 29: 461-462.

3. Alexander MSM, Dias PS, Uttley D. Spontaneous subarachnoid hemorrhage and negative cerebral panangiography: review of 140 cases. J Neurosurg 1986, 64: 537-542.

4.Beguelin C, Seiler R. Subarachonoid hemorrhage with normal panangiography. Neurosurgery 1983, $13: 409-411$.

5. Biller J, Godersky JC, Adams HP. Management of aneurysmal subarachnoid hemorrhage. Stroke 1988, 19: 1300-1305.

6. Bozzola FG, Gorelick PB, Jensen JM. Epidemiology of intracranial hemorrhage. Neuroimag Clin NAm 1992, 2: 1-10.

7. Brismar J, Sundbarg G. Subarachnoid hemorrhage of unknown origin: prognosis and prognostic factors. J Neurosurg 1985, 63: 349-354.

8. Cioffi F, Pasqualin A, Cavazzani P, Da Pian R. Subarahnoid hemorrhage of unknown origin: clinical and tomographical aspects. Acta Neurochir 1989, 97: 31-39.

9. Giombini S, Bruzzone MG, Plunchino F. Subarachnoid hemorrhage of unexplained cause. Neurosurgery 1988 , 22: 313-316.

10. Hayward RD. Subarachnoid haemorrhage of unknown aetilogy: a clinical and radiological study of 51 cases. J Neurol Neurosurg Psychiatry 1977, 40: 926-931.

11. Nishioka H, Torner JC, Kassel NF, Sahs AI, Goettler LC. Cooperative study of intracranial aneurysms and subarachnoid hemorrhage: a long-term prognostic study. Arch Neurol 1984, 41: 1147-1151.

12. Rinkel GJE, Wijdicks EFM, Vermeulen M, Hageman LH, Tans JTJ, van Gijn J. Outcome in perimesencephalic (nonaneurysmal) subarachnoid haemorrhage: a follow-up study in 37 patients. Neurology 1990, 40: 1130-1132.

13. Rinkel GJE, Wijducks EFM, Hasan D, Kienstra GEM, Franke CI, Hageman LM, Vermeulen M, van Gijn J. Outcome in patients with subarachnoid haemorrhage and angiographic according to pattern of haemorrhage on computed tomography. Lancet 1991, 338: 964-968.

14. Rinkel GJE, Wijdicks EFM, Vermeulen M, Hasan D, Brouwers PJAM, van Gijn J. The clinical course of perimesencephalic nonaneurysmal subarachnoid hemorrhage. Ann Neurol 1991, 29: 463-468.

15. Rinkel GJE, Widjdicks EFM, Vermeulen M, Hasan D, Brouwers PJAM, van Gijn J. Acute hydrocephalus in nonaneurysmal perimesencephalic hemorrhage: evidence of CSF block at the tentorial hiatus. Neurology 1992, 42: 1805-1807. 
16. Ruelle A, Lasio G, Boccardo M, Gottlieb A, Severi P. Long-term prognosis of subarachnoid hemorrhages of unknown etiology. J Neurology 1985, 232: 277-279.

17. Solomon RA, Fink ME. Current strategies for the management of aneurysmal subarachnoid hemorrhage. Arch Neurol 1987, 44: 769-774.

18. Van Gijn J. Subarachnoid hemorrhage. Lancet 1992, 339: 653-655.

19. Van Gijn J, van Dogen KJ, Vermeulen M, Hijdra A. Perimesencephalic hemorrhage: a nonaneurysmal and benign form of subarachnoid hemorrhage. Neurology 1985, 35: 493-497.

20. Vermeulen M, van Gijn J. The diagnosis of subarachnoid haemorrhage. J Neurol Neurosurg Psychiatry 1990, 53: 365-372.

21. Watanbe AT, Mackey JK, Lufkin RB. Imaging diagnosis and temporal appearance of subarachnoid hemorrhage. Neuroimag Clin N Am 1992, 2: 53-59. 